\title{
Barriers to accessing TB clinics among Myanmar TB patients attending a Thailand-Myanmar border hospital: a qualitative approach
}

Theint Theint Lwin, Tawatchai Apidechkul and Jongkon Saising School of Health Science, Mae Fah Luang University, Chiang Rai, Thailand Panupong Upala

Center of Excellence for the Hill tribe Health Research, Mae Fah Luang University, Chiang Rai, Thailand, and

Ratipark Tamornpark

School of Health Science, Mae Fah Luang University, Chiang Rai, Thailand

\begin{abstract}
Purpose - This qualitative approach study aimed to understand the barriers to accessing a tuberculosis (TB) clinic in a Thai hospital as experienced by TB patients from Myanmar living on the Thailand-Myanmar border.

Design/methodology/approach - Twenty-two participants were asked to provide information. In-depth interviews were used to gather the information. Each interview lasted $40 \mathrm{~min}$.

Findings - TB patients from Myanmar experience several barriers to accessing TB treatment and care at Mae Sai Hospital, such as language and economic problems, although they are very satisfied with the quality of service and positive attitude of the health care providers. A long waiting time and lack of explanation of the pathogenesis of $\mathrm{TB}$ were noted as negative aspects by the patients and their relatives. The medical staff at the TB clinic were negatively affected by the excessive workload and unsuitability of some methods or technologies. Using budgetary subsidies from agencies to fund TB care and treatment was not sustainable. Foreign TB patients are not subsidized by the national universal insurance scheme of Thailand, and sending TB patients back to their home country is sometimes unavoidable.

Originality/value - Thailand and Myanmar should strengthen their collaboration and develop a system to improve the quality of TB patient care and management for those who are living in poverty and lack education, by focusing on reducing language and economic barriers to accessing health care services including support for medicines and laboratory materials related to TB case management among these populations.
\end{abstract}

Keywords Tuberculosis, Accessibility, Treatment and care, Myanmar, Thailand

Paper type Research Paper

(C) Theint Theint Lwin, Tawatchai Apidechkul, Jongkon Saising, Panupong Upala and Ratipark Tamornpark. Published in Journal of Health Research. Published by Emerald Publishing Limited. This article is published under the Creative Commons Attribution (CC BY 4.0) licence. Anyone may reproduce, distribute, translate and create derivative works of this article (for both commercial and noncommercial purposes), subject to full attribution to the original publication and authors. The full terms of this licence may be seen at http://creativecommons.org/licences/by/4.0/legalcode

The authors would like to thank the Center of Excellence for the Hill Tribe Health Research, Mae Fah Luang University, Thailand in support of the grant (No.26/2019). They would also like to thank the director of Mae Sai Hospital.
Barriers to

tuberculosis service access

Received 30 March 2020 Revised 15 June 2020 12 July 2020

Accepted 13 August 2020 
JHR

36,2

\section{Introduction}

Tuberculosis (TB) is one of the most important human bacterial infections, and health care systems worldwide invest large sums to address the problem [1]. In 2019, the World Health Organization (WHO) estimated that 10.4 million and 558,000 individuals suffered from TB and drug-resistant TB, respectively [1]. TB is prioritized as a first-line human threat in many countries, particularly in the developing world, including Thailand and Myanmar [2]. WHO also reported that approximately 5,000 of 32,000 TB deaths in 2017 were of individuals infected with TB and HIV in Myanmar [3]. However, there is no information available on TB and HIV specific to the Shan State. People who live in rural areas with poor education and low economic status are at a relatively much greater risk of TB infection [4]. Early detection and prompt treatment with a standard regimen are needed to eliminate the disease [5]. The accessibility of health care services is the most important factor in the reduction of TB in all populations. The WHO defines accessibility according to three dimensions: physical accessibility, economic accessibility (affordability) and information accessibility [6]. These barriers were supported by Dhavan et al. [7], particularly in the significance of feasibility and effectiveness of the process and treatment outcomes among migrant workers who live in at a poor socioeconomic status.

In 2019, the Chiang Rai Public Health Office reported more than 1,300 confirmed new TB cases in Chiang Rai Province, and $20 \%$ of the cases were identified in foreigners [8]. Among the foreigners with TB, 80\% were from Myanmar [8]. People from Myanmar who live along the border, especially in Shan State, face challenges in receiving treatment for TB from their local hospitals, and hence sometimes they cross the border to receive treatment at Mae Sai Hospital in Thailand, which is located less than $3 \mathrm{~km}$ from the border [8]. The hospital provides services based on the standard of care for all people, including TB care and treatment [5]. Mae Sai Hospital, according to its primary mission and function, provides health care services for the Thai population [9].

Providing medical care for a person regardless of their race, color, language and nationality is the primary mode of operation of the standard health care service in Thailand. Mae Sai Hospital has more than 200 outpatient visits every day [10]. Approximately $30-50 \%$ of the outpatients are from Myanmar [10], and some of them are diagnosed with active TB; 150-200 new TB cases are detected every year $[11,12]$. Under the agreement that has been made by hospitals in Thailand and Myanmar, there are three main options for the management of TB patients from Myanmar at Mae Sai hospital [8]: referred back to their local hospital, admission as inpatients to start an intensive treatment regimen or patients from Myanmar who are diagnosed with TB can be asked to stay somewhere in Thailand to undergo follow-up at a TB clinic. TB treatment and care is a generally lengthy process and is expensive. To achieve the stated goal of the United Nations regarding the elimination of TB by 2030 [13], all stakeholders need to make improvements, including hospitals located on borders where there is a high prevalence of TB.

This study aimed to identify barriers to accessing TB diagnosis and treatment at a Thai hospital experienced by foreign TB patients living along the border between Thailand and Myanmar.

\section{Methodology}

A qualitative approach was used to elicit information from the participants, who were purposively selected from three groups: TB patients, patients' relatives and health care providers at Mae Sai Hospital. Mae Sai Hospital is located in East Chiang Rai Province, which borders Tachileik City, Shan State, Myanmar. A 17-item questionnaire was developed based on information obtained from a literature review. The validity of the questionnaire was assessed via the item objective congruence (IOC) method [14, 15] and the validity was 
determined by 3 experts in the field: a medical doctor, a nurse working in a TB clinic and an epidemiologist. The questionnaire was tested for feasibility in a pilot study with 10 foreign TB patients at Mae Chan Hospital in April 2019.

Approval for access to Mae Sai Hospital was granted by the hospital director. All relevant medical staff received explanations of study. All participants were purposively selected for interviews, which were conducted in private and confidential rooms by two researchers who were experts in qualitative research: one Thai researcher and medical doctor from Myanmar who was a research team member. The selection of the TB patients for the study was preliminarily based on having been diagnosed with TB disease from a medical doctor and receiving treatment and care in Mae Sai Hospital. All Myanmar TB patients (participants) were selected based on having made at least two visits to a TB clinic at the hospital and a willingness to provide information. Researchers approached patients who attended the TB clinic every Thursday between May to August 2019 until they completed gathering information.

Appointments were made one week in advance, and participants were asked to sign the informed consent form before the interview. The TB patients and their relatives from Myanmar who could not communicate in Thai were interviewed by the researcher from Myanmar. Interviews were conducted from May to August 2019. All interviews were audiotaped after obtaining approval from the participants. Brief notes or field notes were also taken during the interview. Each interview lasted $40 \mathrm{~min}$.

All tapes were transcribed and translated into English. The completeness of each interview was ascertained before the data were subjected to the next step. The interviews were reviewed by all research team members and compared with the notes taken during the interviews. Typed interview transcripts were sent back to the interviewee who was asked to validate the accuracy of the content before further analysis. Data were extracted and analyzed by themes using the NVivo program (version 11). After the thematic analysis was completed, the information was discussed among the members of the research team who determined the findings and drew conclusions.

\section{Ethical issue}

This study was approved by the Mae Fah Luang University Research Ethics Committee on Human Research (No. REH-61215).

\section{Results}

A total of 22 participants provided information for the study (10 TB patients from Myanmar, 5 of their relatives and 7 health care providers). Ten TB patients from Myanmar (five females and five males) were from rural areas in Tachileik District, Shan State, Myanmar, and none of them had health insurance. They were aged between 19 and 64 years, were married and had a primary level of education. Five relatives who participated in the study were middle-aged and employed. Seven health care providers from the Thai hospital participated in the study (one administrator: a hospital director; five individuals who provided direct patients care: two physicians, one public health professional, one nurse, one pharmacist; and one support staff member: medical laboratory technician).

\section{Reasons for obtaining TB treatment at a Thai hospital}

Several reasons for obtaining treatment from a Thai hospital were indicated by the TB patients from Myanmar: a lack of satisfaction with the medical services in hospitals in
Barriers to tuberculosis service access 
JHR

36,2

Myanmar, a lack of medical doctors in hospitals in Myanmar and confidence in the quality of medical services provided by Thai hospitals.

A 64-year-old female said the following:

Approximately three weeks before we had to go to the Mae Sai Hospital, I was admitted to Tachileik Hospital for 2 weeks. The hospital could not give me a diagnosis or treatment, and I was not feeling better. Moreover, I had to wait too long to get test results in Tachileik Hospital. (PT\#1).

A 46-year-old male TB patient said the following:

Every time I visited a hospital for treatment in Myanmar, there was no doctor in the hospital. The nurse told me that the doctor went to a meeting. Last month, I had a cough and fever; my boss advised me to obtain treatment at Mae Sai Hospital. (PT\#2)

A 43-year-old female TB patient said the following:

I have heard about the quality of the health services provided by Mae Sai Hospital from my friends and relatives, so I decided to come here ... I am very happy here. I have received very good care from all the hospital staff, the same as Thai TB patients. (PT\#3).

A 22-year-old relative of PT\#2 said the following:

I had many feelings while my father received treatment at this hospital. I feared the transmission of the disease to my children, so I moved them to another place. I always remember that I have to take my father to get the treatment. In addition, I believe that by coming here to this hospital, he will get the best service. (RT\#2)

A 59-year-old health care provider said the following:

We have a strong and clear policy regarding providing all services to all people in the same way that is not affected by their race, nationality, or economic status. However, given the overcrowding of clients on some days or in some clinics, the patients may need to wait in a long queue before seeing a doctor; we have tried to improve this matter. (HCP\#1)

A 47-year-old health care provider said the following:

I have worked for this clinic for almost 17 years, and we have enough drugs and materials to provide care in our TB clinic; however, the most important issue is that we need more staff to make sure that we have worked through the standard guidelines for TB treatment and control. And it would be helpful to have occasional training sessions. Then we can provide most effective care for our patients. (HCP\#4)

\section{Barriers to accessing TB treatment in Mae Sai hospital}

Two factors were found to be significant barriers to accessing care at this Thai hospital: language and economic barriers. Mae Sai Hospital has hired many support staff in different departments, including TB clinics, to help with communication between health care providers and patients. However, given the large volume of patients each day, the language was found to be the major barrier to accessing medical services at this Thai hospital. The hospital has several options with regard to providing support to patients who have a financial problem, such as waiving all or some medical expenses, allowing them to pay later and offsetting costs with support from nonprofit organizations. However, all Thai TB patients do not face this issue because they are supported by the government through the universal insurance scheme.

Language barriers are common among Myanmar TB patients attending Thai hospitals. A 53-year-old male TB patient said the following:

I have a big problem communicating with health care providers here, even when I have a translator to help me. I do not completely believe that the translator really understood all my problems. I cannot 
speak Thai; I have many questions regarding my health problems, but it is difficult asking questions of my doctor. (PT\#9)

A 47-year-old health care provider said the following:

We have hired staff who are fluent in both Thai and Burmese to help our TB clinic staff communicate with the patients. It is really helpful! However, TB patients do not only come from Myanmar. TB patients of other ethnicities also come, which is a problem because we could not find people for hire who could speak many languages. (HCP\#4)

Economic barriers are also common among TB patients from Myanmar who access Thai hospitals, due to the loss of daily income, the inability to pay for transportation expenses and medical costs

A 64-year-old female TB patient said the following:

It affects our income. When I was admitted to Mae Sai Hospital, I used all my money to pay for medical care here; it costs approximately 1,000 baht. I am worried now; my daughter has to work hard to get money to help me. (PT\#1)

A 37-year-old male TB patient stated the following:

I cannot work now because I have to stay at the hospital. I traveled around Tachileik district for business. At the moment, I cannot do that, and I have lost my income. When I had TB, I could not travel, and I lost my job and money. Fortunately, I have received support from my mother to buy medicine and to cover traveling expenses to the hospital. PT\#6.

A 46-year-old male TB patient said the following:

As I am working in the private sector, I cannot get leave. When I came to the hospital, my salary was cut for leaving. Moreover, I spent approximately 1000 baht hiring the translator when I came to the hospital. I had financial problems because I am the head of my family. I have to pay all medical expenses including the drugs. It costs approximately 1000 baht per month. PT\#2

A 24-year-old male relative of PT\#2 said the following:

When I knew my father had TB, I did not feel anything because we did not know about TB. Since we cannot speak Thai, we had a lot of trouble accessing the hospital. In addition, at the beginning of treatment, we could pay the money for the TB drugs; later, my family experienced a financial burden because the TB treatment was very long. (RT\#2)

A 59-year-old health care provider said the following:

We do have several options for subsidizing medical expenses for foreign patients, including TB patients. In previous years, all TB drugs and materials for laboratory investigations have been covered by the Ministry of Public Health, Thailand. This support covered both Thai and foreign TB patients; however, we have been informed that the financial support for these items is no longer supported by the government. Fortunately, we still have some extra financial plans and support for the TB program to subsidize foreign TB patients, and the support is considered on a case-by-case basis. HCP\#1

\section{Perception and expectation of health care services in Thai hospitals among Myanmar}

TB patients

Eight of 10 TB patients from Myanmar reported high levels of satisfaction with their access to medical services in this Thai hospital while receiving TB treatment and reported receiving the same services as Thai TB patients. A long waiting time and getting little time to have a conversation with a doctor were mentioned as issues that needed improvement. In addition to

Barriers to tuberculosis service access

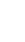


JHR

36,2

providing information regarding the side effects of the drug and the importance of treatment adherence, patients also wanted health care providers to give them other essential information regarding $\mathrm{TB}$, such as the cause of the disease, routes of transmission and prevention methods.

A 47-year-old female said the following:

I have met my doctor twice; he is a very good person. I have been given drugs in a plastic bag and received instructions to take them in a certain way from a nurse. Therefore, I clearly know the drugs that I am to take and when. I am also grateful to the nurses for arranging for me to easily take the drugs ... My doctor told me that all my family members needed to be screened for TB. However, they were not infected. I am very happy. (PT\#10).

A 53-year-old male TB patient said the following:

As you can see, the wait time is so long. When I suffered dizziness at the start of the treatment, I could not sit very well. We had to wait for two hours or more before seeing a doctor. (PT\#9)

Most TB patients spoke about a lack of individual health education about TB, including its transmission, prevention and control. Health care providers provided information only about the potential side effects of the drugs and the importance of continued adherence and treatment completion.

A 46-year-old male TB patient said the following:

The doctor told me that I have a TB, and the disease can be cured if I take the full TB treatment. I have been treated here for 4 months, but I do not know about TB, such as how it is transmitted and how it can be prevented. During the treatment, a nurse explained to me that I must take the TB drugs regularly and gave me some information about the side effects of the TB drugs. (PT\#2)

TB patients from Myanmar are concerned about receiving services from the hospital, and they want to receive information about the pathogenesis of the disease. Moreover, with the economic constraints experienced by the people living in rural Myanmar, the desire to be supported with regard to receiving medical services was commonly expressed by the patients.

A 43-year-old female TB patient said the following:

It would be better if the health care staff could provide more health education about TB, including its transmission, prevention, and control, to TB patients and their families. It would be better for TB patients from Thailand and elsewhere if no one was charged for TB treatment. I want to meet and speak with my doctor about how I am feeling at every follow-up visit, and I want him to pay attention to monitoring my treatment. If he could give me sufficient time to discuss my problems, it would be great. (PT\#3)

A 27-year-old female relative of PT\#8 said the following:

My husband has been diagnosed twice with TB; it was recurrent. When my husband was diagnosed with recurrent TB, I was afraid of it being transmitted to my children. I lived in a village that is located 100 miles from Tachileik. We felt so tired after traveling to the Mae Sai Hospital to take the TB drugs. I want the health care staff to explain how to protect my family from TB. A doctor said that we all had to be screened for TB. It was a large financial burden. (RT\#8)

A 29-year health care provider said the following:

I have 2 years of experience at this TB clinic. We take care of 20-30 TB patients, both Thai and foreign, with 5-6 new patients every week. Even though we have the latest technology to diagnose $\mathrm{TB}$, GeneXpert, it takes time before obtaining the results, approximately 1-2 months. It is not 
practical, and clinical signs, sputum examinations, and chest X-rays are the methods that we use. I feel like the number of new TB cases from Myanmar increase every day. I also am not sure how much they know about the disease. We also do not have time to give them information about the pathogenesis of the disease. (HCP\#2)

A 35-year-old health care provider said the following:

We have many options for managing TB patients from Myanmar; however, sending them back to Myanmar makes me feel bad. I never know what the final treatment outcome is among those sent back to Myanmar. I feel worried about this point. We should have a system to connect the two countries to monitor TB patients. We do not have time to talk with every single person about the pathogenesis of the disease. (HCP\#3)

\section{Discussion}

TB patients in Myanmar who live along the Thailand-Myanmar border prefer to visit Thai hospitals because of the quality of the health care services and attitude of the health care providers. After a diagnosis of TB is made, there are many options regarding how to manage $\mathrm{TB}$, because a long time (6 months to 2 years) is needed to complete the full course of treatment and management is expensive. TB patients face language and economic barriers to accessing TB clinics. Some patients had experienced access to TB care in both Myanmar and Thai hospitals. A long waiting time due to the excessive number of patients attending the hospital was one critical issue mentioned with regard to patient satisfaction. In addition to providing information on drugs and their side effects, including the need to take the drugs regularly and have good adherence to the treatment regimen, health care providers need to give patients more information about the pathogenesis of the disease.

Language was mentioned as a major barrier to accessing Thai hospitals by TB patients from Myanmar. This was supported by several studies in different settings. Nakiwala et al. [16] reported that a language barrier was found in TB clinics in many countries, including developed and developing countries, and posed a major challenge to eliminating TB globally. A systematic review in sub-Saharan Africa also reported that language was identified as one of the major barriers to accessing TB clinics [17]. Moreover, a qualitative study demonstrated the presence of a language barrier in TB clinics in Mozambique [18]. Additionally, it has been demonstrated that language was defined as one of the significant barriers to accessing medical care by TB patients who lived in Thailand-Myanmar border areas [19].

Most TB patients from Myanmar and their relatives reported that economic status was a significant concern when attending Thai hospitals. Due to the long treatment course for $\mathrm{TB}$ and transportation costs, financial concerns constituted one of the major barriers to accessing TB clinics, particularly with regard to adherence to completing the course of treatment. This is supported by a study in Uganda that reported that financial problems were a significant barrier for patients attending the TB clinics [20]. A qualitative study in China also showed that economic factors were identified as a barrier to attending the TB clinics [21]. A systematic review also showed that the financial problems of TB patients posed a major barrier to accessing TB clinics [22]. However, in the study setting, it would be suitable to have a health insurance program that is possible to buy in one country but that can be used in another country.

A long waiting time and the lack of information about the pathogenesis of the disease were mentioned as points for improvement by patients and relatives who attended this hospital in Thailand. To improve the quality of and satisfaction with the service, the 
JHR

36,2

hospital needs to develop a concrete plan, taking into account the limited health resources of the hospital. The continuous improvement of services and encouragement of health care providers to regularly obtain training to update their skills and knowledge related to TB care and treatment should be priorities for the executive manager of the hospital. Overcrowding of patients and long waiting times significantly affect patient satisfaction, and improvements of these issues would lead to increased positive TB treatment outcome rates [17]. A study in China demonstrated that treatment and prevention and control measures at the family and community levels had greater impacts on reducing TB problems, in particular providing information about the pathogenesis of the disease to patients and their relatives [23].

Some limitations to this study were found when validating details of the typed documents with the Myanmar participants, because some of them had returned to their home country, making it difficult to contact them after the process of typing up the audio tapes was completed. This made the period of completing the study four months (May to August 2019) when the confirmation of the information was received from the participants before further analysis. Furthermore, two of the Myanmar TB patients were not Burmese but were from the Akha minority living on the Myanmar side, but because they could speak Thai, the process of interview and confirmation of information was completed in the Thai language.

\section{Conclusion}

The number of TB cases is increasing, particularly in this hospital located along the Thailand-Myanmar border. People who are living in poverty and have a poor education are very vulnerable to TB. However, their access to health care services is affected by several barriers, including language and economic barriers. To eliminate TB, both the Thailand and Myanmar health agencies should develop a concrete plan to work together, such as by providing adequate financial supporting to this particular clinic. These health care providers also need to receive regular training with regard to the updated technology and guidelines to work effectively in this setting. Hiring additional staff who can speak the local languages would help improve the effectiveness of care and treatment. A proper education method for improving knowledge on TB prevention and care at family- and community levels is highly significant, particularly regarding in the use of local languages that is comprehnsible for TB patients from Myanmar.

Conflict of Interest: None

\section{References}

1. World Health Organization [WHO]. Global tuberculosis report 2019. [cited 2019 October 18]. Available from: https://www.who.int/tb/publications/factsheet_global.pdf?ua $=1$.

2. Centers for Disease Control and Prevention [CDC]. Tuberculosis (TB): fact sheets. [cited 2019 October 18]. Available from: https://www.cdc.gov/tb/publications/factsheets/general/ tb.htm.

3. World Health Organization [WHO]. Addressing TB-HIV co-infection. [cited 2020 July 12]. Available from: https://www.who.int/myanmar/activities/addressing-tb-hiv-coinfection.

4. Erlinger S, Stracker N, Hanrahan C, Nonyane S, Mmolawa L, Tampi R, et al. Tuberculosis patients with higher levels of poverty face equal or greater costs of illness. Int J Tuberc Lung Dis. 2019; 23(11): 1205-12. doi: 10.5588/ijtld.18.0814. 
5. Gilpin C, Korobitsyn A, Migliori GB, Raviglione MC, Weyer K. The World Health Organization standards for tuberculosis care and management. Eur Respir J. 2018; 51(3): 1800098. doi: 10.1183/ 13993003.00098-2018.

6. World Health Organization [WHO]. Gender, equity, human right: accessibility. [cited 2019 November 7]. Available from: https://www.who.int/gender-equity-rights/understanding/ accessibility-definition/en/.

7. Dhavan P, Dias HM, Creswell J, Weil D. An overview of tuberculosis and migration. Int J Tuberc Lung Dis. 2017; 21(6): 610-23. doi: 10.5588/ijtld.16.0917.

8. Laingoen O, Apidechkul T, Upala P, Tamornpark R, Foungnual C, Paramee R. Cost-effectiveness of TB treatments to Burmese and Laotian patients by Thai hospitals on the border with Myanmar and Laos. J Health Res. 2020; 34(1): 13-21. doi: 10.1108/Jhr-01-2019-0011.

9. Mae Sai Hospital. Vision and missions. [cited 2019 October 18]. Available from: http://www. maesaihospital.com/page_a.php?cid=13.

10. Mae Sai Hospital. Annual report 2019. [cited 2019 October 18]. Available from: http://www. maesaihospital.com/page_b.php?cid $=23 \&$ cname $=\% \mathrm{~A} 2 \% \mathrm{E} 8 \% \mathrm{D} 2 \% \mathrm{C} 7 \% \mathrm{BB} \% \mathrm{C} 3 \% \mathrm{D} 0 \% \mathrm{AA} \%$ $\mathrm{D} 2 \% \mathrm{CA} \% \mathrm{D} 1 \% \mathrm{C} 1 \% \mathrm{BE} \% \mathrm{D} 1 \% \mathrm{~B} 9 \% \mathrm{~B} 8 \% \mathrm{EC}$.

11. Mae Sai Hospital. Integrated plan for TB clinic. [cited 2019 October 18]. Available from: http:// www.maesaihospital.com/page_bx.php?cid $=18 \&$ cname $=\& c n o=946 \&$ cno $2=\&$ show $=10$.

12. Tschirhart N, Sein T, Nosten F, Foster AM. Migrant and refugee patient perspectives on travel and tuberculosis along the Thailand-Myanmar Border: a qualitative study. PLoS One. 2016; 11(8): e0160222. doi: 10.1371/journal.pone.0160222.

13. World Health Organization [WHO]. The end TB strategy. [cited 2019 December 3]. Available from: https://www.who.int/tb/End_TB_brochure.pdf?ua =1.

14. Turner RC, Carlson L. Indexes of item-objective congruence for multidimensional items. Int J Test. 2003; 3(2): 163-71. doi: 10.1207/S15327574IJT0302_5.

15. Sanitya R, Sinjindawong S. Design of students' evaluation of teaching: the case of Sripatum University. Int J Inf Educ Technol. 2017; 7(5): 367-73. doi: 10.18178/ijiet.2017.7.5.896.

16. Nakiwala D, Kellgren L, Herzmann C, Olaru ID, Brown M, Ferrand R, et al. Language discordance between tuberculosis patients and healthcare providers challenging universal access. Eur Respir J. 2017; 49(4): 1700116. doi: 10.1183/13993003.00116-2017.

17. Sullivan BJ, Esmaili BE, Cunningham CK. Barriers to initiating tuberculosis treatment in subSaharan Africa: a systematic review focused on children and youth. Glob Health Action. 2017; 10(1): 1290317. doi: 10.1080/16549716.2017.1290317.

18. De Schacht C, Mutaquiha C, Faria F, Castro G, Manaca N, Manhiça I, et al. Barriers to access and adherence to tuberculosis services, as perceived by patients: a qualitative study in Mozambique. PLoS One. 2019; 14(7): e0219470. doi: 10.1371/journal.pone.0219470.

19. Tschirhart N, Nosten F, Foster AM. Access to free or low-cost tuberculosis treatment for migrants and refugees along the Thailand-Myanmar border in Tak province, Thailand. Int J Equity Health. 2016; 15(1): 100. doi: 10.1186/s12939-016-0391-z.

20. Cattamanchi A, Miller CR, Tapley A, Haguma P, Ochom E, Ackerman S, et al. Health worker perspectives on barriers to delivery of routine tuberculosis diagnostic evaluation services in Uganda: a qualitative study to guide clinic-based interventions. BMC Health Serv Res. 2015; 15: 10. doi: 10.1186/s12913-014-0668-0.

21. Hutchison C, Khan MS, Yoong J, Lin X, Coker RJ. Financial barriers and coping strategies: a qualitative study of accessing multidrug-resistant tuberculosis and tuberculosis care in Yunnan, China. BMC Public Health. 2017; 17(1): 221. doi: 10.1186/s12889-017-4089-y.

22. Yang WT, Gounder CR, Akande T, De Neve JW, McIntire KN, Chandrasekhar A, et al. Barriers and delays in tuberculosis diagnosis and treatment services: does gender matter? Tuberc Res Treat. 2014; 2014: 461935. doi: 10.1155/2014/461935.

\section{Barriers to tuberculosis service access}


23. Chen B, Liu M, Gu H, Wang X, Qiu W, Shen J, et al. Implementation of tuberculosis infection control measures in designated hospitals in Zhejiang Province, China: are we doing enough to prevent nosocomial tuberculosis infections?. BMJ Open. 2016; 6(3): e010242. doi: 10.1136/ bmjopen-2015-010242.

\section{Corresponding author}

Tawatchai Apidechkul can be contacted at: tawatchai.api@mfu.ac.th

For instructions on how to order reprints of this article, please visit our website: www.emeraldgrouppublishing.com/licensing/reprints.htm Or contact us for further details: permissions@emeraldinsight.com 